\title{
Deep layer-resolved core-level shifts in the beryllium surface
}

\author{
Aldén, Magnus; Skriver, Hans Lomholt; Johansson, Börje
}

Published in:

Physical Review Letters

Link to article, DOI:

10.1103/PhysRevLett.71.2457

Publication date:

1993

Document Version

Publisher's PDF, also known as Version of record

Link back to DTU Orbit

Citation (APA):

Aldén, M., Skriver, H. L., \& Johansson, B. (1993). Deep layer-resolved core-level shifts in the beryllium surface. Physical Review Letters, 71(15), 2457-2460. https://doi.org/10.1103/PhysRevLett.71.2457

\section{General rights}

Copyright and moral rights for the publications made accessible in the public portal are retained by the authors and/or other copyright owners and it is a condition of accessing publications that users recognise and abide by the legal requirements associated with these rights.

- Users may download and print one copy of any publication from the public portal for the purpose of private study or research.

- You may not further distribute the material or use it for any profit-making activity or commercial gain

- You may freely distribute the URL identifying the publication in the public portal

If you believe that this document breaches copyright please contact us providing details, and we will remove access to the work immediately and investigate your claim 


\title{
Deep Layer-Resolved Core-Level Shifts in the Beryllium Surface
}

\author{
Magnus Aldén, ${ }^{1}$ H. L. Skriver, ${ }^{2}$ and Börje Johansson ${ }^{1}$ \\ ${ }^{1}$ Condensed Matter Theory Group, Physics Department, Uppsala University, S-75121 Uppsala, Sweden \\ ${ }^{2}$ Physics Department, Technical University of Denmark, DK-2800 Lyngby, Denmark
}

(Received 17 June 1993)

\begin{abstract}
Core-level energy shifts for the beryllium surface region are calculated by means of a Green's function technique within the tight-binding linear muffin-tin orbitals method. Both initial- and final-state effects in the core-ionization process are fully accounted for. Anomalously large energy shifts are found for the three topmost surface layers in agreement with recent experimental data. The reason for this extraordinary behavior is explained.
\end{abstract}

PACS numbers: $71.20 . \mathrm{Ad}, 73.20 . \mathrm{Hb}$

During the last one and a half decades a lot of experimental and theoretical work has been devoted to the study of surface core-level binding energy shifts [1-14]. The largest surface core-level shift is found for samarium metal where it amounts to about $7 \mathrm{eV}$ [1]. The reason for this very large shift is the valence change from trivalency in the bulk to divalent samarium at the surface $[1,3,4]$, and therefore this element cannot be directly compared to other metals. For ordinary elemental metals, however, the size of the surface core-level shifts is well below $1 \mathrm{eV}$. Because of experimental difficulties there have not been any reports on subsurface layer shifts, except for tantalum and tungsten where small shifts were observed for the second surface layer atoms [13]. However, most recently a surface and two subsurface layer shifts have been observed for beryllium [14]. The shifts decrease in magnitude from $-0.82 \mathrm{eV}$ at the surface to $-0.57 \mathrm{eV}$ in the next sublayer and $-0.26 \mathrm{eV}$ in the third surface layer. These shifts are most anomalous-for example, the outermost layer shift is the largest one so far reported for an elemental metal (except for samarium) and the subsurface layer shifts are extremely large. In this respect beryllium represents a unique physical situation.

In this paper we consider beryllium theoretically and investigate to what extent these exceptional experimental data are supported by explicit calculations. For comparison we also report on calculated results for three other hcp crystals; magnesium, scandium, and lutetium. Magnesium is a simple metal as is beryllium, while scandium and lutetium are early $3 d$ and $5 d$ transition metals, respectively.

The theoretical core-level shifts we will present include both initial- and final-state effects. The surface shift is evaluated from first principles by calculating the surface segregation energy of a charge neutral core-ionized atom. This is a direct $a b$ initio application of the theory presented by Johansson and Mårtensson [8], where the central assumption is that the symmetric part of the line profile for the core-level corresponds to a completely screened final state. Similar calculations have recently been performed for surface core-level shifts of transition metals giving results in very good agreement with ex- periments [15]. Therefore the method seems well suited for an accurate investigation of the anomalous beryllium data.

Recent analysis by low-energy electron diffraction (LEED) implies that the surface layer of the $\mathrm{Be}(0001)$ surface is expanded outwardly by $5.8 \%$ relative to the ideal termination of the surface [16]. This result was supported by theoretical findings [17], suggesting a $3.9 \%$ larger separation between the outermost two layers than in the bulk. We thus face a behavior which appears to be quite singular among the elemental metals, since these usually exhibit a contraction of the outermost surface layer. This might suggest that the observed extraordinary core-level energy features in the beryllium surface region originate from effects due to the expanded surface layer. However, it is one of our objectives of this Letter to show that already an unrelaxed beryllium surface displays core-level shifts very similar to those observed experimentally. Therefore the unusual shifts in beryllium do not just originate from distorted bond lengths, but are intrinsic consequences of the valence electron distribution.

We have employed the Green's function technique within the tight-binding linear muffin-tin orbitals method and local-density theory [18] as originally implemented for surfaces and interfaces by Skriver and Rosengaard [19]. The technique has now been further extended to permit calculations of the electronic structure and total energies of a substitutional impurity either in the bulk or at the surface. The formalism rests on the pioneering work by Gunnarsson, Jepsen, and Andersen [20], except that here we utilize the most localized (tight-binding) orbital representation [21]. For the present application it should be stressed that we make a complete separation between the two different physical situations at hand, namely, (a) the bulk impurity problem in a perfect bulk geometry and (b) the surface impurity for a geometry and potential of the real surface. We thus aim at as realistic a description as possible and consider infinite systems rather than its simulation via a suitable slab or supercell. The formalism for the two different cases is completely analogous and is implemented at the 
same level of approximation, thereby enabling an effective cancellation of errors in, e.g., the computed total energies. In the present calculations we employ the frozen core and atomic sphere approximations, but include the dipole contribution to the intersphere potentials. Based on convergence tests, utilizing the generalized phase shift to sum the one-electron energies [20], we use a cluster region consisting of the impurity site plus four shells of nearest neighbor host atoms, corresponding to 55 and 51 atoms for the fcc and hcp crystals, respectively. We have estimated that the convergence in, e.g., shell number of the calculated core-level binding energy shifts is better than $0.02 \mathrm{eV}$. The main limitation of our approach is that it requires an ideal termination of the layer spacings at the surface. Other computational details together with a comprehensive description of the method will be presented elsewhere [22].

The surface core-level shift is thus evaluated by means of separate impurity calculations for the bulk and for the surface, yielding two different impurity-solution energies $E_{\text {imp }}^{\text {bulk }}$ and $E_{\text {imp }}^{\text {surface }}$, respectively. The impurity is a neutral core-ionized atom where an electron has been removed from a core level $[\mathrm{Be}(1 s), \operatorname{Mg}(2 p), \mathrm{Sc}(2 p)$, and $\mathrm{Lu}(4 f)]$. The surface core-level shift, for surface layer $L$, may be obtained as the layer-resolved surface segregation energy, i.e.,

$$
\Delta_{c}^{L}=E_{\mathrm{imp}}^{\mathrm{surface} ; L}-E_{\mathrm{imp}}^{\mathrm{bulk}},
$$

where the layer index $L$ denotes the layer in which the core-ionized impurity has been located in the surface impurity calculation. With the presently used sign convention, a positive shift corresponds to a higher binding energy for the surface core level than for the bulk core level.

The results for the most close-packed surface of hcp beryllium and hypothetical fcc beryllium are given in Fig. 1. A large negative shift in the core-level binding energy

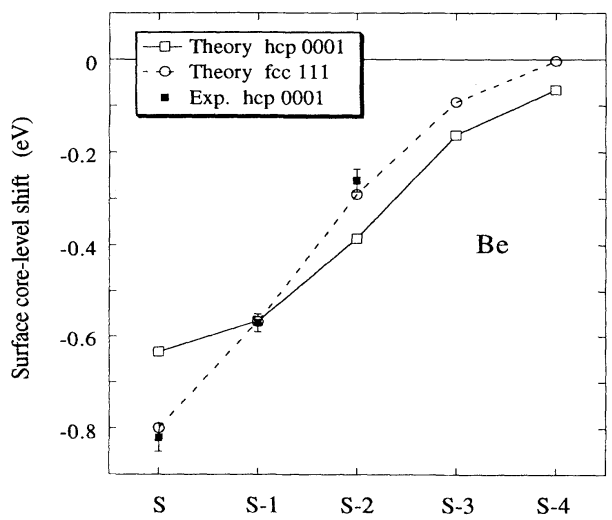

FIG. 1. Comparison between calculated (open symbols) and experimental [14] (solid squares with error bars) layerresolved surface core-level shifts. $\mathrm{S}$ denotes the surface layer, S-1 the first subsurface layer, etc. is found for the surface layer, which is only slightly reduced in the second surface layer, and then smoothly decreasing towards zero but still significant even as far down into the bulk as the fourth surface layer. This behavior is found for both the hcp 0001 and fcc 111 surfaces, which is indeed reasonable since they have a similar packing but with different stacking. As can also be seen in Fig. 1 the agreement between the layer-resolved experimental data and our $a b$ initio results is qualitatively very good. Thus the present calculations are consistent with the observed extreme behavior of the core-level energies in the beryllium surface region and its interpretation as due to layer-resolved surface energy shifts.

In a detailed comparison between experiment and theory, it has to be remembered that the zero temperature calculations had to be performed for an unrelaxed surface layer, while experimentally the outmost surface layer distance is expanded by $5.8 \%$ [16]. Clearly the discrepancy seen in Fig. 1 between experiment and our hcp 0001 calculations could be due to this difference. However, it is most striking that the computed data for the fcc 111 surface give a complete agreement with experiment. This raises the possibility that at the surface domain beryllium has reconstructed into an fcc surface; i.e., the standard $A B A B$ hcp stacking sequence has turned into an $A B C$ fcc layer sequence at the surface. It is not clear to us if this possibility has been considered in the analysis of the LEED data [16] or not, and we leave this as an open question. There might also be another reason for the good agreement between data and our fcc 111 calculations. In bulk Be the $c / a$ ratio is 1.567 , while the expanded surface layer is more close to the ideal packing ratio 1.63. Thus the outermost layer distance fits rather well to the corresponding distance for the fcc 111 surface, which might explain the agreement in Fig. 1 [23].

To identify the reason for these extraordinary beryllium surface shifts we shall compare with calculated shifts for the hcp metals magnesium, scandium, and lutetium. The calculated results for these elements are given in $\mathrm{Ta}-$ ble I together with experimental surface layer shifts for $\mathrm{Mg}$ [6] and $\mathrm{Lu}$ [7]. Unfortunately, these data are taken from polycrystalline samples and cannot therefore be directly compared to our calculated values. Still the agree-

TABLE I. Calculated surface (S) and subsurface (S-1, S-2, S-3) core-level shifts for the hcp 0001 surface of $\mathrm{Mg}$, Sc, and $\mathrm{Lu}$. The experimental (Exp) shifts refer to polycrystalline samples.

\begin{tabular}{lccccc}
\hline \hline Metal & \multicolumn{5}{c}{ Core-level shift (eV) } \\
& $\mathrm{S}-3$ & $\mathrm{~S}-2$ & $\mathrm{~S}-1$ & $\mathrm{~S}$ & Exp (S) \\
\hline $\mathrm{Mg}$ & -0.011 & -0.034 & 0.010 & 0.188 & $0.14^{\mathrm{a}}$ \\
$\mathrm{Sc}$ & -0.007 & -0.027 & 0.139 & 0.261 & \\
$\mathrm{Lu}$ & -0.015 & -0.054 & -0.056 & 0.513 & $0.7^{\mathrm{b}}$ \\
\hline \hline
\end{tabular}

${ }^{\mathrm{a}}$ See Ref. [6]. $\quad{ }^{\mathrm{b}}$ See Ref. [7]. 
ment appears to be satisfactory. Especially we notice the change of sign between $\mathrm{Be}$ and $\mathrm{Mg}$, which is remarkable in view of the fact that these two elements belong to the same column in the periodic table. This very pronounced difference is well reproduced by our calculations demonstrating the accuracy of our method. In Fig. 2 we compare the computed variation of the layer-resolved shifts relative to the outermost layer shift. For the simple metal magnesium the magnitude of the shifts is heavily reduced in the subsurface layers and even exhibits an oscillatory behavior as one proceeds into the bulk. A similar rapid decrease of the shifts is also found for the $5 d$ transition metal lutetium. For the $3 d$ metal scandium, however, the core-level shift of the first subsurface layer is only reduced by $\sim 50 \%$ relative to the surface layer value. It is evident that scandium metal in this respect behaves much more similar to beryllium than to magnesium and lutetium.

To analyze these results we first consider the nature of the valence electrons in the different metals. For the divalent metals beryllium and magnesium the orbitals involved are $2 s 2 p$ and $3 s 3 p$, respectively, and for trivalent scandium and lutetium the corresponding quantities are $4 s 3 d$ and $6 s 5 d$. The screening charge of a core hole will essentially consist of an electron in a $p$ state (Be and $\mathrm{Mg}$ ) or in a $d$ state ( $\mathrm{Sc}$ and $\mathrm{Lu}$ ). Since the $p$ orbitals are more extended than the $d$ orbitals, this suggests surface core-level shifts for a larger number of surface layers for the simple metals than for the transition metals. However, as can be seen from Fig. 2 there is no essential difference between $\mathrm{Mg}$ and $\mathrm{Lu}$ in this respect. Instead there is another circumstance which plays a much more predominant role for the layer extension of the shifts.

This is the fact that the screening orbitals for $\mathrm{Be}(2 p)$ and $\mathrm{Sc}(3 d)$ are such that there are no $p$ or $d$ states in the respective core region, against which they would have to orthogonalize. This makes the $2 p(3 d)$ charge extra

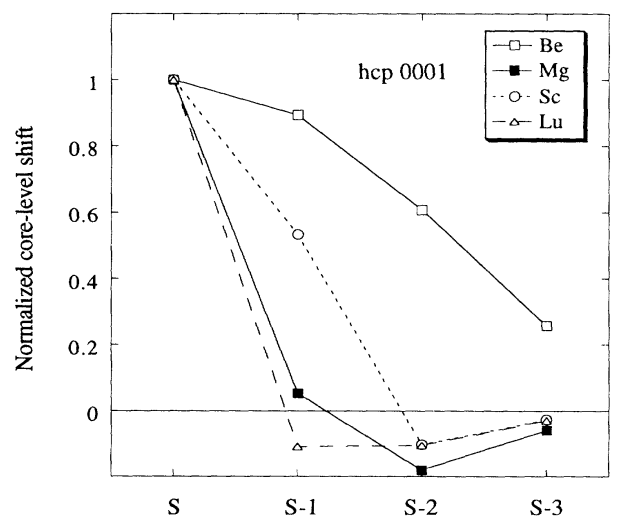

FIG. 2. Comparison between calculated layer-resolved shifts, normalized to that of the surface layer (S), for the hcp 0001 surface of $\mathrm{Be}$ (open squares), $\mathrm{Mg}$ (solid squares), $\mathrm{Sc}$ (circles), and $\mathrm{Lu}$ (triangles). S denotes the surface layer, S-1 the first subsurface layer, etc. vulnerable to a disturbance in the core region or to a disturbance from the immediate surroundings of the atom like the presence of a surface. It is for this reason that beryllium displays large core-level shifts in the surface region and this is also likely to be the reason for its abnormal surface layer expansion. Scandium is similarly affected (Fig. 2) although here the core-level shifts after the second layer become very small, due to the relatively contracted nature of the $3 d$ orbital.

In conclusion, we have from explicit calculations demonstrated that different classes of metals can show widely different behavior of the core-level energies in the surface region. Transition metals show only core-level energy shifts in at most two surface layers while the simple metal beryllium behaves differently and shows shifts for as many as four layers. The reason for this is that the $2 p$ orbital for beryllium is less rigid than, for example, the $3 p$ orbital for magnesium, since the latter has to be orthogonal to the $2 p$ core level imposing a restriction on its spatial distribution. Therefore changes in the immediate surroundings of a beryllium atom give larger effects on the distribution of the $2 p$ charge than for the $3 p$ charge in magnesium, explaining the vast difference in the surface core-level shift behavior between these two simple metals.

We would like to thank I. A. Abrikosov and N. M. Rosengaard for discussions of various aspects of the Green's function techniques. M.A. and B.J. are grateful for financial support from the Göran Gustafsson Foundation and the Swedish Natural Science Research Council. The work of H.L.S. was supported by grants from the Novo Nordisk Foundation and the Danish Research Councils through the Center for Surface Reactivity.

[1] G.K. Wertheim and G. Creselius, Phys. Rev. Lett. 40, 813 (1978).

[2] P.H. Citrin, G.K. Wertheim, and Y. Baer, Phys. Rev. Lett. 41, 1425 (1978).

[3] B. Johansson, Phys. Rev. B 19, 6615 (1979).

[4] J.K. Lang and Y. Baer, Solid State Commun. 31, 945 (1979).

[5] M.C. Desjonqueres, D. Spanjaard, Y. Lasailly, and C. Guillot, Solid State Commun. 34, 807 (1980).

[6] R. Kammerer, J. Barth, F. Gerken, C. Kunz, S.A. Flodström, and L.I. Johansson, Phys. Rev. B 26, 3491 (1982).

[7] J. Schmidt-May and R. Nyholm, in $V U V$-Radiation Physics, edited by A. Weinreb and A. Ron [Ann. Isr. Phys. Soc. 6, 348 (1983)].

[8] B. Johansson and N. Mårtensson, Phys. Rev. B 21, 4427 (1980); Helv. Phys. Acta 56, 405 (1983).

[9] P.H. Citrin and G.K. Wertheim, Phys. Rev. B 27, 3176 (1983).

[10] N. Mårtensson, H.B. Saalfeld, H. Kuhlenbeck, and M. Neumann, Phys. Rev. B 39, 8181 (1989).

[11] A.M. Begley, R.G. Jordan, W.M. Temmerman, and P.J. Durham, Phys. Rev. B 41, 11780 (1990).

[12] A. Flodström, R. Nyholm, and B. Johansson, in $A d$ - 
vances in Surface and Interface Science, Volume 1: Techniques, edited by R.Z. Bachrach (Plenum Press, New York, 1992).

[13] J.F. van der Veen, P. Heiman, F.J. Himpsel, and D.E. Eastman, Solid State Commun. 37, 555 (1981); 38, 595 (1981).

[14] L.I. Johansson, H.I.P. Johansson, J.N. Andersen, E. Lundgren, and R. Nyholm, preceding Letter, Phys. Rev. Lett. 71, 2453 (1993); (private communication).

[15] M. Aldén, H.L. Skriver, and B. Johansson (to be published).

[16] H.L. Davis, J.B. Hannon, K.B. Ray, and E. W. Plummer, Phys. Rev. Lett. 68, 2632 (1992).

[17] P.J. Feibelman, Phys. Rev. B 46, 2532 (1992).

[18] S.H. Vosko, L. Wilk, and M. Nusair, Can. J. Phys. 58, $1200(1980)$
[19] H.L. Skriver and N.M. Rosengaard, Phys. Rev. B 43, 9538 (1991); 46, 7157 (1992).

[20] O. Gunnarsson, O. Jepsen, and O.K. Andersen, Phys. Rev. B 27, 7144 (1983).

[21] O.K. Andersen and O. Jepsen, Phys. Rev. Lett. 53, 2571 (1984); O.K. Andersen, Z. Pawlowska, and O. Jepsen, Phys. Rev. B 34, 5253 (1986).

[22] M. Aldén, I.A. Abrikosov, H.L. Skriver, N.M. Rosengaard, and B.Johansson (to be published).

[23] In an attempt to estimate the effects of surface relaxation, by means of properly relating the calculated total energies of a $c / a$ expanded hcp 0001 surface system to those of unexpanded bulk, we could deduce only a small effect (less than $50 \mathrm{meV}$ ). The same result holds if the shift is calculated for an expanded hcp lattice both in the bulk and at the surface. 